\title{
Isolation of New Delhi metallo-ßeta- lactamase- I (NDM-I) producing klebsiella pneumoniae in a Neonate in Kwazulu-Natal, South Africa
}

\begin{abstract}
Isolation of New Delhi Metallo-ßeta- lactamase-1(NDM-1) producing Klebsiella pneumoniae in a neonate in KwaZulu-Natal, South Africa. Carbapenems have been a last line of defense in the treatment of infections caused by antimicrobial-resistant Enterobacteriaceae. The emergence of resistance in Enterobacteriaceae mediated via carbapenemases is a major concern as these isolates show resistance to antibiotics commonly used to treat Gram-negative infections. The first documented case of NDM-1producing Enterobacteriaceae in a neonate within 24 hours of birth is reported here. This is a case report of a neonate with New Delhi Metallo-Beta-lactamse-1 producing Klebsiella pneumoniae in Kwazulu Natal South Africa. Emergence of this organism is worrisome in the neonatal unit, since treatment options are restricted. Active surveillance is required to prevent spread of this organism. Strict infection control and rationale use of antimicrobials is required, to prevent acquisition of these organisms.
\end{abstract}

Volume 2 Issue 4 - 2015

\author{
Radhika Singh,' Prasha Mahabeer, ${ }^{2}$ Koleka \\ Mlisana, ${ }^{2}$ Kimesh Naidoo,' Prashini Moodley ${ }^{3}$ \\ 'Department of Pediatrics, University of Kwazulu Natal, South \\ Africa \\ ${ }^{2}$ Department of Medical Microbiology, Kwazulu- Natal Academic \\ Hospital, South Africa \\ ${ }^{3}$ Department of Infection control and prevention, University of \\ Kwazulu Natal, South Africa
}

Correspondence: Radhika Singh, Department of Pediatrics, N.R. Mandela School of Medicine, University of Kwazulu Natal, South Africa, Email Singhr2@ukzn.ac.za

Received: May 20, 2015 | Published: July 30, 2015

\section{Introduction}

Multi-drug resistant Gram negative rods are a common cause of nosocomial infections. The widespread use of broad spectrum agents for these infections has led to the emergence of Multi-drug resistant gram-negative (MDRGN) organisms and the emergence and spread of novel $\beta$-lactamases. These carbapenemases have been isolated from a variety of gram negative bacilli and continues to escalate amidst a limited antimicrobial pipeline.

Infections due to Gram-negative organisms are increasingly being reported from neonatal units and many outbreaks amongst neonates described over the past 10 years have isolated Klebsiella pneumonia as the causative agent. A recent outbreak of Klebsiella pneumoniae occurred in the neonatal nursery of a large teaching hospital in Durban, Kwazulu Natal (KZN), during which a Carbapenemase producing gram negative bacillus was isolated. We describe the first documented neonatal case of NDM-1 producing Klebsiella pneumoniae in South Africa.

\section{Case report}

A male neonate was born at term to a 24 year old HIV infected woman by caesarean section. The mother had a CD4 count of 523 cells/ $\mathrm{mm}^{3}$ and was on nevirapine (NVP) as part of the PMTCT (prevention of mother to child transmission) guidelines. The indication for caesarean section was fetal distress with meconium staining of the liquor. Post-delivery, the APGAR scores were recorded as 8 and 9 at 1 and 5 minutes respectively. The neonate appeared stable and on transfer to the nursery after oral suctioning, he developed respiratory distress requiring ventilator support shortly post-delivery and an endotracheal aspirate on intubation was obtained. The neonate was commenced on first line antibiotics which included Benzyl penicillin and Gentamycin.

There were no radiological features of meconium aspiration syndrome on admission. On day 3 the chest $\mathrm{x}$-ray (CXR) showed bilateral infiltrates in keeping with bronchopneumonia. A repeat septic work up was done including a lumbar puncture and a repeat endotracheal aspirate. The $\mathrm{C}$-reactive protein (CRP) was noted to be elevated $(35 \mathrm{mg} / \mathrm{ml})$ and the cerebrospinal fluid was normal. Due to an ESBL positive (Extended spectrum Beta Lactamase positive) Klebsiella pneumoniae outbreak in nursery during that period, antibiotics were changed to Meropenem since all the Klebsiella pneumoniae isolated during that period were susceptible to Meropenem.

The neonate was extubated successfully on day 4 of life. The results of the endotracheal aspirate taken on intubation showed a Klebsiella pneumoniae resistant to Meropenem and susceptibleto Colistin. As the neonate was extubated and a follow up CRP was normal on day 5 , a decision was not to treat. However on day 6 the neonate showed clinical deterioration and was commenced on Colistin at $40000 \mathrm{iu} / \mathrm{kg} / \mathrm{dose} 8 \mathrm{hrly}$ for 7 days. The neonate was isolated and subsequently made a good clinical recovery. He was discharged home on day 14. Screening of the mother and the environmental cultures were negative. The minimum inhibitory concentration (MIC) of this isolate for Meropenem was $4 \mathrm{mg} / 1$ on the Vitek-2 system and this was confirmed by E-Test. The isolate was sent to a reference laboratory for molecular testing. Molecular characterization was performed using the Light Cycler 480 (Roche Applied Science, Germany) and this revealed an NDM-1 producing Klebsiella pneumoniae.

\section{Discussion}

This is the first documented neonatal case of NDM-1 producing Klebsiella pneumoniae in South Africa causing early onset infection. There have been cases of NDM-1producing Klebsiella pneumoniae reported from adult patients. Carbapenemase producing Klebsiella pneumoniae amongst neonates have also recently been reported from South America and Asia. In KZN, there has been a steady increase in the MIC (minimal inhibitory concentration) trends in the susceptibility profiles of Klebsiella pneumonia associated with outbreaks. Most outbreaks in our setting are the result of nosocomial spread of ESBL producing K.pneumoniae strains. (Unpublished 
data). Although the organism was isolated from a single patient and was not associated with the outbreak, the implication of the isolation of an organism displaying multi-drug resistance to conventional antimicrobial agents is worrisome. This comes in the wake of ignored warnings from protagonists of antibiotic stewardship programmes. Carbapenems like Meropenem and Imipenem are used to treat severe gram negative infections. Their use has increased with the increased prevalence of ESBL producing Gram negative organisms. The recent emergence of Carbapenem resistant Enterobacteriaceae (CRE) is worrisome; since antimicrobial treatment options are very restricted. Resistance to these agents poses a serious threat to our ability to treat life-threatening infections especially in neonates and infants.

Carbapenem-resistant Gram-negative bacteria is a worldwide clinical problem that is principally associated with the production of carbapenemases. ${ }^{2}$ There are several mechanisms of carbapenem resistance including decreased outer-membrane permeability, efflux pumps and the production of carbapenem-hydrolyzing $\beta$-lactamases (carbapenemases). ${ }^{3}$ It is the acquired carbapenemases that are more serious as they are carried on plasmids and can spread among different strains and species of bacteria. ${ }^{3}$ They belong to different molecular classes and include "Imipenem-hydrolyzing $\beta$-lactamase (IMP), Verona integron-encoded Metallo- $\beta$-lactamase (VIM), New Delhi Metallo- $\beta$-lactamase-1 (NDM-1), Klebsiella pneumoniae carbapenemases (KPC) and OXA-48. ${ }^{3}$ New Delhi Metallo- $\beta$ lactamase (NDM-1) is a carbapenemase that confers resistance to all $\beta$-lactam antibiotics, except Aztreonam. NDM-1 was first identified in Klebsiella pneumoniae and Escherichia coli recovered from a Swedish patient who was transferred from India and Pakistan in 2008. ${ }^{4,5}$ Govind et al. ${ }^{6}$ recently reported the first documented case of NDM 1 Enterobacter cloacae in KZN with an epidemiological link to the Indian subcontinent. Since then, other cases in several hospitals have been identified. These plasmid-carrying NDM are highly transmissible to other bacteria.

Bacteria carrying NDM can colonize the gastrointestinal systems of humans for prolonged periods and can spread through contaminated water sources and environmental surfaces. In KZN, the detection of the isolated case of NDM-1 Klebsiella pneumoniae in a neonate is probably the tip of the iceberg. There have been many case reports in adult patients. Active surveillance looking for CRE (Carbapenem resistant Enterobacteriaceae) colonization may help define the magnitude of the problem. However, this may be difficult in a resource limited setting with insufficient resources and infrastructure. Vigilance is also required from the laboratory side as detection of these isolates requires a high index of suspicion as they may only have small reductions in carbapenem susceptibility. ${ }^{3}$
Currently, the only effective way to reduce the further development and spread of CREs is the implementation of strict infection prevention coupled with rational use of antimicrobials. These are both dependent on human behavior and may not be easily implemented. Unless new effective antimicrobial agents are developed, the mortality from extensively and pan drug resistant GNBs will continue to increase in neonates. In summary, this case report highlights the emergence of carbapenase resistant Enterobacteriaceae. The organism was cultured on day 1 of life from the endotracheal aspirate indicating early onset infection. Though the blood culture was negative, we opted to treat it, due to increasing $\mathrm{C}$-reactive protein and clinical deterioration. One needs to implement and follow strict infection control policies, to prevent further spread of the organism, since treatment options are minimal.

\section{Acknowledgments}

None.

\section{Conflicts on interest}

The authors declare that there are no conflicts of interest.

\section{Funding}

None.

\section{References}

1. Nordmann P, Cuzon G, Naas T. The real threat of klebsiella pneumoniae carbapenemase-producing bacteria. Lancet Infect Dis. 2009;9(4):228236

2. Sidjabat H, Nimmo GR, Walsh TR, et al. Carbapenem resistance in Klebsiella pneumoniae due to the New Delhi metallo-beta-lactamase. Clin Infect Dis. 2011;52(4):481-484.

3. Advice on carbapenemase producers: recognition, infection control and treatment: advisory committee on antimicrobial resistance and healthcare associated infections (ARHAI) UK.

4. Nordmann P, Poirel L, Walsh TR, et al. The emerging NDM carbapenemases. Trends Microbiol. 2011;19(12):588-595.

5. Kumarasamy KK, Toleman MA, Walsh TR, et al. Emergence of a new antibiotic resistance mechanism in India, Pakistan, and the UK: a molecular, biological, and epidemiological study. Lancet Infect Dis. 2010;10(9):597-602.

6. Govind CN, Moodley K, Peer AK, et al. NDM-1 imported from India first reported case in South Africa. S Afr Med J. 2013;103(7):476-478 